\title{
ESCALAS TERMOQUÍMICAS E ESPECTROSCÓPICAS DE BASICIDADE: BREVE REVISÃo
}

Robson Fernandes de Farias*

Departamento de Química, Universidade Federal de Roraima, CP 167, 69301-970 Boa Vista - RR

Recebido em 5/12/01; aceito em 26/8/02

THERMOCHEMICAL AND SPECTROSCOPIC BASICITY SCALES: A BRIEF REVIEW. A brief review about the main thermochemical and spectroscopic basicity scales is presented, with a discussion about the range of reliability of them. Is concluded that, as a general trend, thermochemical basicity scales are more sensitives, and so reliable, than spectroscopic ones.

Keywords: thermochemistry; spectroscopy; basicity scales.

\section{INTRODUÇÃO}

O conceito de ácidos e bases constitui-se num dos mais fundamentais em Química, tendo um grande poder na predição e racionalização de reações ${ }^{1}$. As definições do que sejam um ácido ou uma base podem ser as mais diversas, com as definições de Arhenius, Bronsted-Lowry e Lewis estando entre as mais conhecidas e empregadas, uma vez que se aplicam grandemente às reações em solução. A despeito das diferentes definições existentes, verifica-se que, em geral, estas definições se sobrepõem e/ou englobam, conforme ilustrado pelo diagrama de Jensen².

Contudo, para que o conceito de ácidos e bases possa ser de utilidade mais efetiva na racionalização das reações químicas, tornase necessário o estabelecimento de dados quantitativos, ou seja, precisa-se saber o quão "forte" ou "fraco" seja um determinado ácido ou uma determinada base. Mas, como se pode medir a "força" de um determinado ácido ou base?

O procedimento geralmente utilizado é medir-se a variação de uma determinada propriedade, quando da reação de diferentes bases de Lewis com um certo ácido, que será definido como a espécie receptora de elétrons, e será sempre o mesmo em todas as reações. Contudo, espera-se que uma determinada ordem de basicidades seja mantida, independentementre da escala utilizida (embora isso nem sempre se verifique, como veremos mais adiante).

No estabelecimento quantitativo da basicidade de espécies doadoras de par(es) de elétrons (bases de Lewis), o uso da calorimetria e da espectroscopia têm se mostrado de grande utilidade. Discutem-se aqui as principais escalas de basicidade elaboradas com base em dados termoquímicos (calorimétricos) ou espectroscópicos. Para efeito de comparação/discussão, os valores de basicidade para um grupo de quinze substâncias, em algumas das escalas consideradas, são apresentados na Tabela 1. Vale a pena ressaltar que existem várias outras escalas, além das aqui apresentadas, como as escalas espectroscópicas propostas por Munakata ${ }^{3}$ e Abraham ${ }^{4}$.

O presente trabalho tem por finalidade apresentar uma breve revisão sobre as principais escalas termoquímicas e espectroscópicas de basicidade, discutindo-se as vantagens e limitações de cada uma.

\section{ESCALAS ESPECTROSCÓPICAS DE BASICIDADE}

Sandström e colaboradores ${ }^{5,6}$ utilizaram-se de dados de espectroscopia na região do infravermelho para o estabelecimento

*e-mail: robsonfarias@aol.com
Tabela 1. Valores de basicidade para quinze moléculas selecionadas, em cada uma das escalas consideradas para discussão

\begin{tabular}{lccccc} 
composto & $\mathrm{D}_{\mathrm{S}}$ & $\mathrm{Cu} \lambda_{\text {máx. }}$ & $-\Delta \delta$ & $\mathrm{D}_{\mathrm{N}}$ & $\mathrm{BF}_{3}$ \\
\hline benzeno & 9 & - & - & 0,1 & - \\
nitrometano & 9 & 532 & - & 2,7 & 37,6 \\
nitrobenzeno & 9 & 533 & - & 4,4 & 35,8 \\
acetona & 15 & 571 & 0,72 & 17 & 76,0 \\
thf & 17 & 579 & 0,65 & 20 & 90,4 \\
água & 17 & 591 & 0,54 & 18 & - \\
metanol & 18 & - & - & 19 & - \\
dmf & 24 & 602 & 1,22 & 26,6 & 110,5 \\
dma & 24 & - & 1,28 & 27,8 & 112,1 \\
dmso & 27,5 & 613 & 1,25 & 29,8 & 105,3 \\
tmu & 24 & - & - & 29,6 & 108,6 \\
anilina & 34 & 570 & - & 35 & $-\overline{10}$ \\
piridina & 38 & 613 & - & 33,1 & 128,1 \\
2-pic & 39 & - & - & 39 & 123,4 \\
4-pic & 39 & - & - & - & 134,2 \\
\hline
\end{tabular}

thf $=$ tetraidrofurano $; \mathrm{dmf}=$ dimetilformamida $; \mathrm{dma}=$ dimetilacetamida; $\mathrm{dmso}=$ dimetilsufóxido; 2-pic $=2$-picolina $(2$-metilpiridina); 4-pic $=4$-picolina

de uma escala de basicidades, apresentando o chamado $\mathrm{D}_{\mathrm{S}}$ "donor strength", que teria um valor diferente para cada base, ou seja, seria uma medida da "força" desta base. Tal escala foi estabelecida levando-se em conta a diferença na freqüência vibracional de estiramento do $\mathrm{HgBr}_{2}$ "livre" no estado gasoso, e quando complexado com uma determinada base, conforme expresso pela equação abaixo, e esquematicamente representado na Figura 1:

$\mathrm{D}_{\mathrm{S}}=v \mathrm{HgBr}_{2}$ (gas) $-v \mathrm{HgBr}_{2}(\mathrm{sol})$

Neste caso, o $\mathrm{HgBr}_{2}$ foi escolhido como ácido em função de ser estável (não sofrer dissociação) propiciando, assim, a interação com substâncias com grande força de coordenação, tais como aminas e fosfinas, mantendo sua unidade estrutural, e formando compostos do tipo $\mathrm{HgBr}_{2} . \mathrm{L}_{2}$ de estrutura pseudotetraédrica. L é uma molécula doadora de elétrons, monodentada (possui um único sítio doador de elétrons).

Sone e Fukuda ${ }^{7-9}$ propuseram uma escala de basicidade a partir do deslocamento da banda de absorção na região do visível $\left(\mathrm{Cu} \lambda_{\text {máx. }}\right)$ do complexo $[\mathrm{Cu}(\mathrm{tmen})(\mathrm{acac})]^{+}(\mathrm{tmen}=$ tetrametiletilenodiamina; 


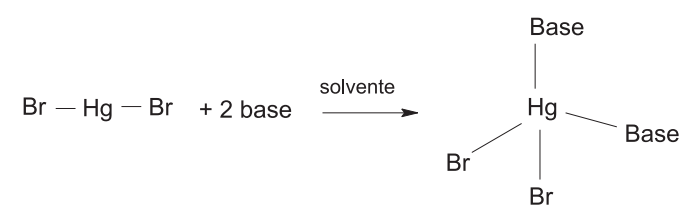

Figura 1. Representação esquemática da reação considerada para o estabelecimento do valor de $D_{S}$ Sendo $v_{1}$ a freqüência de estiramento da ligação $\mathrm{Hg}-\mathrm{Br}$ no $\mathrm{HgBr}_{2}$ gasoso e $v_{2}$ a freqüência de estiramento da mesma ligação após reação do $\mathrm{HgBr}_{2}$ com as moléculas do solvente (uma base L), formando um composto pseudotetraédrico de fórmula $\mathrm{HgBr}_{2} \cdot L_{2}, v_{1}-v_{2}=\Delta v=D_{S}$

acac $=$ acetilacetonato), quando solvatado por diferentes solventes (utilizando-se o solvente puro). Assim, tem-se uma escala de basicidade estabelecida com base em dados de espectroscopia na região do ultravioleta-visível (UV-VIS).

$\mathrm{Na}$ escala de Sone e Fukuda explora-se o seguinte fenômeno: um íon $\mathrm{Cu}^{2+}$ "livre" apresenta a configuração $\left(\mathrm{t}_{2 \mathrm{~g}}\right)^{6}\left(\mathrm{e}_{\mathrm{g}}\right)^{3}$ (elétrons d). Devido ao efeito estabilizador dos dois ligantes quelantes, o "buraco" nos orbitais e estará localizado no orbital $\mathrm{d}_{\mathrm{x}}^{2}{ }^{2} \mathrm{y}_{\mathrm{y}}^{2}$, isto no complexo não solvatado, formando-se ligações mais fortes no plano equatorial dos ligantes. Assim, o átomo de cobre possui dois sítios receptores de elétrons na direção axial (orbital $\mathrm{d}_{\mathrm{z}}^{2}$ ). A energia deste orbital aumentará à medida que aumente o poder dos ligantes axiais (base cuja "força" será medida), causando um "deslocamento para o azul", que pode ser acompanhado utilizando-se espectroscopia na região do UV-VIS. A Figura 2 ilustra a variação na diferença de energia entre os orbitais $t_{2 g}$ e e quando da formação do complexo com a base desejada. Para efeito de comparação, basta destacar que o "número doador do solvente" (DN), estabelecido com base na escala de Sone e Fukuda tem os valores 4 para o nitrobenzeno, com o qual forma um composto violeta-avermelhado, 17 para a acetona (composto violeta-azulado), 27 para a formamida (composto azul) e 51 para soluções extremamente básicas de piridina (composto verde).

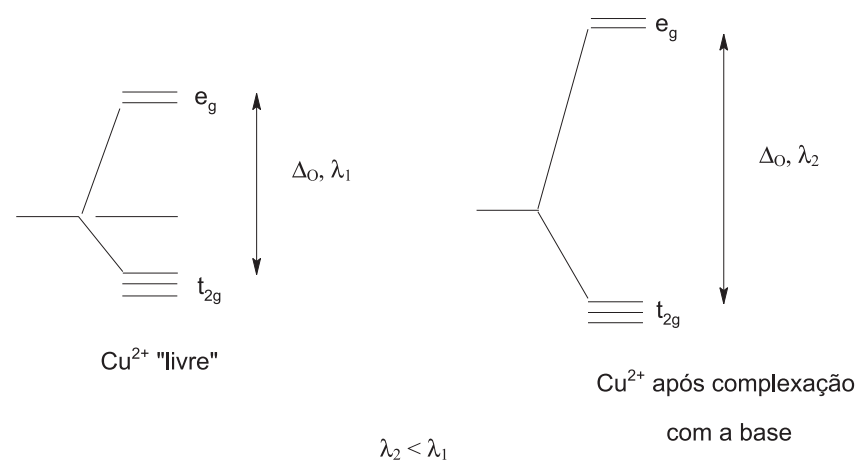

Figura 2. Representação esquemática da variação de energia entre os níveis $t_{2 g}$ e $e_{g}$, antes e após reação do $\mathrm{Cu}^{2+}$ com a base cuja "força" será medida na escala de Sone e Fukuda

Hahn e colaboradores ${ }^{10}$ estabeleceram uma escala de basicidades para substâncias contendo nitrogênio ou oxigênio como átomos doadores, com base em dados de ressonância magnética nuclear protônica, RMN ${ }^{1} \mathrm{H}$. Nesta escala, o valor de $-\Delta \delta\left(\mathrm{CHCl}_{3}\right)$, que é o deslocamento químico do clorofórmio puro (no espectro de RMN $\left.{ }^{1} \mathrm{H}\right)$ com relação ao clorofórmio como soluto numa solução diluída, tendo como solvente a base considerada, é uma medida da basicidade. Quanto maior (mais negativo) $\Delta \delta\left(\mathrm{CHCl}_{3}\right)$ mais forte é a base.

\section{ESCALAS TERMOQUÍMICAS DE BASICIDADE}

Drago $^{11,12}$ estabeleceu também uma escala de basicidades com base em dados termoquímicos, resumida na equação: $-\Delta H=E_{A} E_{B}+$ $\mathrm{C}_{\mathrm{A}} \mathrm{C}_{\mathrm{B}}$, onde os índices $\mathrm{A}$ e $\mathrm{B}$ referem-se ao ácido e à base, respectivamente, com os fatores $\mathrm{E}$ e $\mathrm{C}$ associando-se, respectivamente, às contribuições eletrostáticas e covalentes para a entalpia de reação do par A-B. Esta escala não será aqui discutida, uma vez que existe, neste caso, uma "interpenetração" entre os valores de basicidade e acidez, com os valores de $\mathrm{E}_{\mathrm{B}}$ e $\mathrm{C}_{\mathrm{B}}$ variando, dependendo do ácido considerado. Nas próprias palavras de Drago, "as outras escalas são unidimensionais, a minha é bidimensional”.

Sem dúvida, uma das mais famosas escalas de basicidade estabelecidas a partir de dados termoquímicos foi proposta por Gutmann $^{13}$. Tal escala baseia-se na entalpia da reação entre um ácido, no caso $\mathrm{SbCl}_{5}$, e uma determinada base, em solução de 1,2dicloroetano, formando um aduto de estequiometria 1:1. O chamado número doador, $\mathrm{D}_{\mathrm{N}}$, uma medida da força da base, seria simplesmente o valor da entalpia de reação multiplicado por -1. Gutmann escolheu o $\mathrm{SbCl}_{5}$ como ácido de referência pelas seguintes características: formação de adutos de estequiometria 1:1 com todas as moléculas ligantes; apresentar energias de hibridização similares para todos os compostos formados, em função de idênticas transformações estruturais sofridas em todos os casos (de bipiramidal para octaedro distorcido); ser um ácido forte, garantindo rápida formação de um aduto, mesmo em presença de bases fracas e, finalmente, pelo fato da ligação Sb-Cl não ser facilmente hidrolizável, evitando-se assim interações indesejáveis com as espécies doadoras.

Um escala termoquímica mais moderna, e também mais precisa (consegue detectar pequenas variações nos valores de basicidade) foi proposta por Maria e $\mathrm{Gal}^{14}$. Esta escala, estabelecida para 75 substâncias não-protônicas, consiste na medida da entalpia de reação $(\mathrm{kJ}$ $\mathrm{mol}^{-1}$ ) entre a base considerada e um ácido previamente escolhido, no caso $\mathrm{BF}_{3}$, em uma solução de diclorometano, de acordo com a reação: $\mathrm{BF}_{3}(\mathrm{~g})+: \mathrm{Base}(\mathrm{sol}) \rightarrow \mathrm{Base}: \mathrm{BF}_{3}(\mathrm{sol})$. Quanto maior (mais exotérmico) o valor da entalpia de reação, mais "forte" é considerada a base. A medida da basicidade neste caso seria o valor da entalpia de reação multiplicada por $-1 . \mathrm{BF}_{3}$ foi escolhido como ácido por considerarem os autores que as interações $\mathrm{BF}_{3} /$ soluto(base) $/ \mathrm{CH}_{2} \mathrm{Cl}_{2}$ seriam, neste caso, proporcionais à basicidade do soluto. Assim, embora as interações com o solvente não possam ser evitadas, garante-se que as mesmas afetarão proporcionalmente todas as moléculas estudadas, estabelecendo-se uma escala relativa de basicidades.

\section{DISCUSSÃO}

Como podemos evidenciar, com base nas cinco escalas de basicidade apresentadas, o estabelecimento de tais escalas começa pela escolha de um ácido "ideal", ou seja, uma substância capaz de se comportar como ácido (ácido de Lewis, nas escalas consideradas), e que apresente de preferência um único sítio de coordenação (ou sítios equivalentes, caso haja mais de um), garantindo-se assim que todas as bases consideradas se coordenarão ao ácido pelo mesmo ponto, o que evita variações nos valores de basicidade que não sejam decorrência unicamente da "força" da base. A base, por sua vez, deve ser também monodentada, pela mesma razão que o ácido deve apresentar um único sítio de coordenação. Além disso, caso as reações ácido-base sejam efetuadas em solução, torna-se necessário escolher um solvente que interaja o mínimo possível, quer com o ácido, quer com a base, uma vez que o objetivo é medir a força da interação entre ambos, e não de ambos com o solvente.

Comparando-se os dados da Tabela 1, podemos comprovar que nenhuma escala de basicidades é "perfeita", não apenas por não se- 
rem completas, mas também por não serem capazes de distinguir, em termos de basicidade, duas substâncias diferentes. Como norma geral, verifica-se que as escalas termoquímicas de basicidade são mais "sensíveis" que as escalas espectroscópicas. Assim, enquanto os valores de $\mathrm{D}_{\mathrm{S}}$ não diferenciam 2-picolina de 4-picolina, a escala com base nos valores de entalpia de reação com $\mathrm{BF}_{3}$ apresenta valores bastante diferentes para as duas substâncias, o mesmo sendo válido para as basicidades do nitrometano e do nitrobenzeno. Considerando-se o conjunto benzeno, nitrometano e nitrobenzeno, verificase que a escala de Gutmann diferencia estas substâncias, enquanto os valores de $\mathrm{D}_{\mathrm{S}}$ apontam para um único valor de basicidade para as três moléculas.

Considerando-se apenas as escalas espectroscópicas, verifica-se que a escala de Hahn, baseada em dados de ressonância magnética nuclear protônica, é mais "sensível" que as escalas baseadas em dados de espectroscopia na região do infravermelho ou do UV-VIS. A escala de Hahn consegue, por exemplo, diferenciar dmf de dma, o que não acontece quanto se utilizam os valores de $\mathrm{D}_{\text {s }}$. Considerandose as moléculas de acetona, thf e água, verifica-se que os valores de basicidade fornecidos por $\Delta \delta$ apontam, aparentemente, para uma ordem de basicidades exatamente oposta à fornecida pelos valores de $\mathrm{Cu} \lambda_{\text {máx. }}$ Contudo, devemos lembrar que quanto maiores os valores de $\lambda$, menor a energia $(E=h c / \lambda$, onde $E$ é energia, h é a constante de Planck, c é a velocidade da luz e $\lambda$ é o comprimento de onda da radiação eletromagnética considerada), associada à transição eletrônica, o que significa dizer que as bases associadas aos maiores valores de $\lambda$ são as mais fracas (provocam um menor desdobramento dos orbitais d, do cobre). As duas escalas, portanto, concordam entre si.

Considerando-se as escalas termoquímicas apenas, podemos constatar que a seqüência de valores de basicidade para dmf, dma e dmso são diferentes nas escalas de Gutmann e $\mathrm{BF}_{3}$, o mesmo sendo válido para o grupo nitrometano, nitrobenzeno e acetona. Assim, uma base mais "forte" numa escala, pode ser mais "fraca" na outra. Contudo, tais fatos tornam apenas evidente a relatividade envolvida no conceito de basicidade, bem como as dificuldades envolvidas no estabelecimento de uma escala que possa ser tida como definitiva (supondo que isto seja possível).

A despeito das considerações precedentes, foi recentemente demonstrado ${ }^{15}$, através de estudo comparativo entre diferentes escalas, que, de um modo geral, as escalas termoquímicas são mais sensíveis (capazes de detectar pequenas variações no valor de basicidade) que as escalas espectroscópicas sendo, portanto, em geral, mais confiáveis. Tal conclusão foi atingida tendo-se por referência os valores de entalpia de reação de diferentes bases frente ao cloreto de zinco ${ }^{15}$, $\mathrm{ZnCl}_{2}$. A título de exemplo, basta citar que as entalpias de reação da dmf e da dma com $\mathrm{SbCl}_{5}, \mathrm{BF}_{3}$ e $\mathrm{ZnCl}_{2}$, apontam sempre para uma maior basicidade da dma, como seria de se esperar, em função da presença de um grupo metil "extra", em comparação com a dmf. Por outro lado, o valor de basicidade para ambas as substâncias é o mesmo, se for considerada a escala espectroscópica estabelecida a partir da reação com $\mathrm{HgBr}_{2}$.

$\mathrm{Na}$ comparação entre as diferentes escalas de basicidade, devemos levar em conta também que outros efeitos, devidos às interações intermoleculares, não são "detectados" pela interação principal, objeto da medida, fato este que termina por determinar diferenças entre os valores de basicidade. Como exemplo típico podemos citar o valor da água na escala de Gutmann ${ }^{13}$, comparando-se os valores calorimétrico e não calorimétrico.

\section{REFERÊNCIAS}

1. Jensen, W. B.; The Lewis Acid-Base Concepts-an Overview, Wiley: New York, 1980.

2. Chagas, A. P.; Quim. Nova 2000, 23, 126.

3. Munakata, M.; Kitagawa, S.; Miyazima, M.; Inorg. Chem. 1985, 24, 1638.

4. Abraham, M. H.; Duce, P. P.; Prior, D. V.; Barrat, D. G.; Morris, J. J.; Taylor, P. J.; J. Chem. Soc., Perkin Trans. 1989, 2, 1355.

5. Sandström, M.; Persson, I.; Persson, P. A.; Acta Chem. Scan. 1990, 44, 653.

6. Persson, I.; Sandström, M.; Goggin, P. L.; Inorg. Chim. Acta 1987, 129, 183.

7. Fukuda, Y.; Sone, K.; Bull. Chem. Soc. Jpn. 1972, 45, 465.

8. Soukup, R. W.; Sone, K.; Bull. Chem. Soc. Jpn. 1987, 60, 2286.

9. Soukup, R. W.; Schimid, R.; J. Chem. Ed. 1985, 62, 459.

10. Hahn, S.; Miller, W. M.; Lichtenthaler, R. N.; Prausnitz, J. M.; J. Solution Chem. 1985, 14, 129.

11. Drago, R. S.; Vogel, G. C.; Needham, T. E.; J. Am. Chem. Soc. 1971, 93, 6014.

12. Drago, R. S.; Wayland, B. B.; J. Am. Chem. Soc. 1965, 87, 3571.

13. Gutmann, V.; The Donor-acceptor Appoach to Molecular Interactions, Plenum Press: New York, 1978.

14. Maria, P.-C.; Gal, J.-F.; J. Phys. Chem. 1985, 89, 1296

15. de Farias, R. F.; Airoldi, C.; J. Therm. Anal. Calorim. 2002, 67, 579. 